\title{
Heat transfer of bubbly flow on inner wall of annular channel
}

\author{
Pavel Lobanov ${ }^{1, *}$, Aleksandr Kurdyumov ${ }^{1}$, and Aleksandr Svetonosov ${ }^{2}$ \\ ${ }^{1}$ Kutateladze Institute of Thermophysics, Novosibirsk, 630090, Acad. Lavrentiev ave., 1, Russia \\ ${ }^{2}$ Novosibirsk State University, Novosibirsk, 630090, Pirogova, 2, Russia
}

\begin{abstract}
Experimental investigations of heat transfer from the heated wall to the two-phase bubbly flow were performed in vertical annular channel using air-water system. The IR-thermography and miniature temperature sensors were used to measure heat transfer coefficients. The influence of bubbles on heat transfer is shown in comparison with the case of single phase flow. The presence of bubbles in the flow leads to heat transfer intensification in the annular channel even for low void fractions.
\end{abstract}

\section{Introduction}

An important problem of creating the energy-efficient equipment is the study of passive heat transfer intensifiers. One of the simplest examples of such equipment is a pipe-in-pipe heat exchanger in which heat is transferred between the flows in the inner and outer pipes and the medium around the outer pipe.

Often the temperature of the medium in such heat exchangers exceeds the critical one that causes boiling of the coolant. This leads to the appearance of vapor bubbles in the flow. Despite a rather large number of studies, a number of questions are still open even for a single-phase flow. Studies of the local structure of the two-phase gas-liquid flow, and especially heat transfer in such flows, are very limited in the literature [1-6].

Therefore, studying the local structure of bubble gas-liquid flow in annular channels as well as the effects of gas bubble addition on heat transfer is a relevant task.

\section{Experimental setup}

The experimental setup was a closed circuit in a liquid and an open circuit in the gas phase. The test liquid, i.e. distilled water, was pumped by the centrifugal pump to the test section of the main tank. The temperature of the liquid was controlled at the level of $25 \pm 0.2^{\circ} \mathrm{C}$. The flow rate was monitored using an ultrasonic flowmeter.

The section, simulating the pipe-in-pipe heat exchanger, was an axisymmetric annular channel (Fig. 1 a). To center the inner tube we used the distancing elements installed to avoid flow disturbance in the measurement area. The hydraulic diameter of the annular

\footnotetext{
*Corresponding author: lobanov@itp.nsc.ru
} 


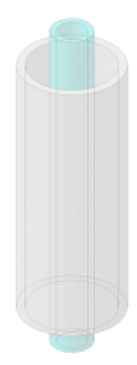

a

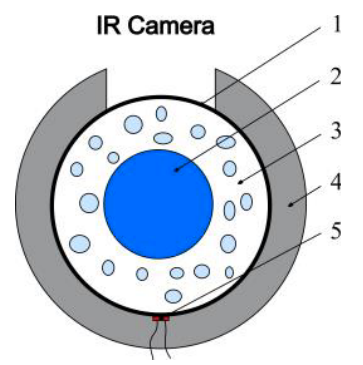

b

Fig. 1. Schematic of the test section: $a$ - annular channel; $b$ - cross section of heat transfer measurement unit: 1 - thin stainless steel wall; 2 - inner pipe; 3 - two-phase flow; 4 thermal insulation; 5 - RTDs.

channel $\left(D_{h}=D-d\right)$ was equal to $22 \mathrm{~mm}$. The length of the flow stabilization area before the measurement region was $80 \mathrm{Dh}$.

The gas flow rate was determined using Bronkhorst (up to $1 \mathrm{l} / \mathrm{min}$ ) and Aalborg (up to 5 $1 / \mathrm{min}$ ) gas flow controllers. The measurement error of liquid and gas flow rates was $\pm 2 \%$ of the measured value.

Generation of bubbles was carried out by feeding air into the liquid flow through the capillaries of $50 \mathrm{~mm}$ length and an internal diameter of $0.7 \mathrm{~mm}$, uniformly distributed in the channel cross-section. Twenty four capillaries were used for the annular channel. Bubbles' size measurements were carried out using video shooting with shadow illumination and subsequent image processing. To reduce optical distortion a box with a square crosssection, filled with immersion liquid, was used.

For experimental studies of heat transfer from the heated wall to the bubble flow, we used the test section, made of thin-walled stainless steel [7] (Fig. 1 b). The inner diameter of the test section was $42 \mathrm{~mm}$, the wall thickness was $0.5 \mathrm{~mm}$, and the heating area length was $500 \mathrm{~mm}$. The section was heated using electric current. Current was supplied using a laboratory power source with power up to $2 \mathrm{~kW}$, used for power control. The source was connected to a step-down transformer, allowing for supply of the alternating current within the range of 300-400 A to the test section at an operating voltage of 2-2.4 V. Current strength was measured using a measuring transformer, and the voltage in the work area was measured with a precision multimer with a measuring error of $0.02 \mathrm{~V}$. Behind the measuring area there was a pipe section $42 \mathrm{~mm}$ in diameter and a length of $1 \mathrm{~m}$ to avoid the output effects.

The infrared thermography was used as a main measuring method. To increase emissivity in thermographic measurements, the external surface of the working area was painted black. For the measurements we used a thermal imager Fluke Ti32. For comparison, 3 thermal resistors Pt1000 were installed on the opposite side, at distances $\mathrm{z}=$ 68,230 and $405 \mathrm{~mm}$ on the surface of the work area. Thermal resistors were pressed against the pipe surface through a thin layer of thermal paste. Around the heat resistors there was insulation to reduce heat loss to the environment (Fig. 1 b). The results obtained by different methods are in good agreement. At the input and output of the work area the control resistance thermometers were set to assess the degree of heating of the working fluid while passing through this section.

In the experiments on heat transfer distribution, voltage was supplied to the work area. After the time needed for the flow stabilization, 20 thermal images were recorded. At the same time, temperatures at the work area input and output, as well as the signals of the temperature sensors, mounted on the surface of the heated wall, were recorded. Given the axial symmetry of the flow, thermal images were processed to vertically average regions, corresponding to the entire height of the work area and the distance from the axis of 


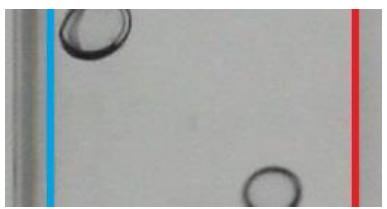

a

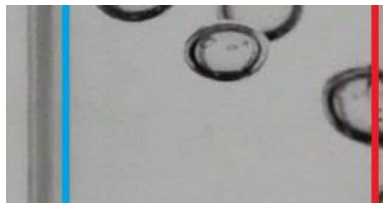

b

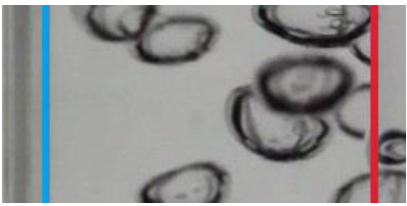

C

Fig. 2. Photographs of bubbles in annular gap. $\operatorname{Re}=7500$. Blue lines - outer wall of the annular channel, red lines - inner one: $a-\beta=0.007 ; b-\beta-0.02 ; c-\beta=0.033$

symmetry of $\pm 20 \%$ from the diameter of the heated tube. For all modes the wall temperature was determined. Using the known heat flux on the wall, temperatures at the input and output of the work area and the field of temperature distribution in the work area, the heat transfer coefficient $\mathrm{h}$ and the Nusselt number - Nu were calculated that was the main result of our experimental studies.

In the experiments, the repeated measurements of the temperature of the work area at the wall without heating were performed (over 100 measurements). The temperature of the liquid according to the temperature sensors, installed in the tank, as well as before and after the measurement section, was $25 \pm 0.1^{\circ} \mathrm{C}$, and the ambient temperature was $\sim 27.5^{\circ} \mathrm{C}$. At that, the deviation of the temperature of the channel wall, determined by means of infrared thermography, was within the range $t_{\mathrm{W}}=25 \pm 0.15^{\circ} \mathrm{C}$. Thus, we can assume that the error of the wall temperature measured with a thermal imager in our case is $\pm 0.15^{\circ} \mathrm{C}$. The measured temperature difference of the liquid flow and the wall depending on the flow regime and position of the measured points was within the range $4-5^{\circ} \mathrm{C}$ that leads to the uncertainty in determining the difference of wall temperature from $\pm 3 \%$ to $\pm 4 \%$.

\section{Results of experiments}

The measurements were carried out at Reynolds numbers of the liquid phase $\operatorname{Re}=\left(u^{*} D_{h} / v\right)$ - 5000-10000, the gas content was up to 3.3\%. Photographs of bubbly flow in the annular channel for different flow conditions are shown in Fig. 2. The outer and inner walls of the channel are shown by colour lines. Equivalent diameter of bubbles in our experiments was within the range $2-5 \mathrm{~mm}$. Small bubbles were observed to tend migrating to the channel walls, while large bubbles moved in the whole volume of the test section.

Thermograms, obtained using IR camera are shown in Fig. 3. One can see that incensement of gas flow rate leads to decrease of the wall temperature. Further processing of presented thermal images and signals of temperature sensors allows us to obtain heat transfer characteristics.

Relative heat transfer intensification at different gas void fractions and $\mathrm{Re}$ is shown in

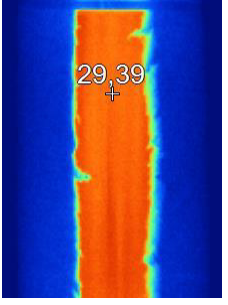

a

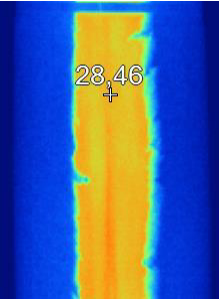

b

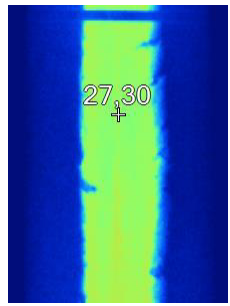

C

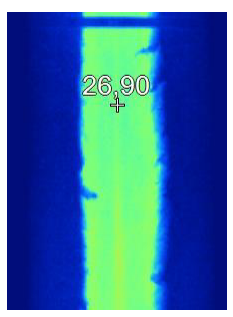

d

Fig. 3. IR images of the outer wall of the annulus: $a$ - single phase flow; $b-\beta=0.007 ; c-\beta-0.02 ; d-\beta=0.033$ 


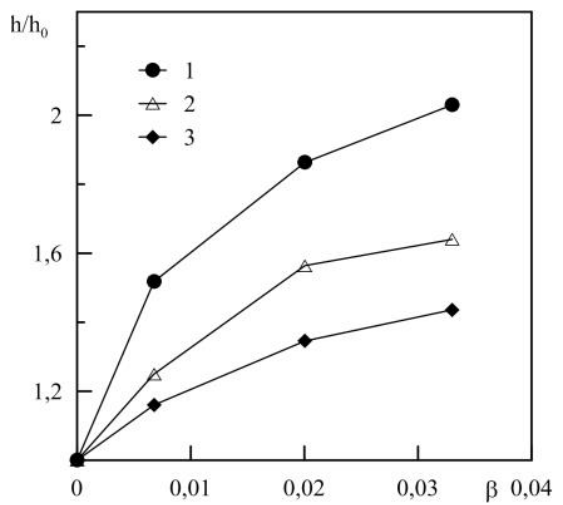

Fig. 4. Heat transfer enhancement in two phase bubbly flow:

$1-\operatorname{Re}=5000 ; 2-\operatorname{Re}=7500 ; 3-\operatorname{Re}=10000$.

Fig. 4. Adding gas bubbles to the liquid flow results in heat transfer enhancement. The increase in gas flow rate leads to an increase in difference for the cases of single-phase and two-phase flows. For $\operatorname{Re}=5000$ and $\beta=3 \%$ heat transfer enhances almost twice, while for $\mathrm{Re}=10000 \beta=3 \%$ the ratio is approximately 1.4 .

Similar results were previously obtained in the IT SB RAS, at investigating the wall shear stress and the local flow structure in bubble flows at comparable Reynolds numbers [5]. It was shown that the intensification of heat transfer occurs due to transformation of velocity profiles of two-phase flows that leads to a substantial increase in the fluid velocity gradient in the near-wall region.

\section{Conclusions}

The study of heat transfer from the heated wall to single- and two-phase flows has been carried out. It is shown that the addition of gas bubbles to the flow leads to the heat transfer intensification. At that, the significance of this effect decreases with increasing Reynolds number of liquid that was reported earlier by other authors. The effects of heat transfer increase due to appearing bubbles should be taken into account in the calculations when adding a dispersed phase in the flow or in the beginning of boiling.

We can conclude that at small Reynolds numbers in the liquid phase, the addition of the dispersed phase is an effective method of heat transfer intensification. However, with increasing Re the effect of bubbles on heat transfer decreases.

This work was supported by the Russian Foundation for Basic Research (project No. 15-38-21040).

\section{References}

1. B. Ozar, J. J. Jeong, A. Dixit, J. E. Juliá, T. Hibiki, M. Ishii, Chem. Eng. Sci. 63, 3998 (2008)

2. T. Hibiki, R. Situ, Y. Mi, M. Ishii, Int. J. Heat Mass Transfer. 46, 427 (2003a)

3. T. Hibiki, R. Situ, Y. Mi, M. Ishii, Int. J. Heat Mass Transfer. 46, 1479 (2003b)

4. R. Situ, T. Hibiki, X. Sun, Y. Mi, M. Ishii, Int. J. Heat Mass Transfer. 47, 5351 (2004)

5. O.N. Kashinskii, A.S. Kurdyumov P.D. Lobanov, Thermophys Aeromech. 1837 (2011) 
6. P.D. Lobanov, M.V. Timoshevskiy, J. Phys.: Conf. Ser. 754, 032013 (2016)

7. O.N. Kashinsky, V.V. Randin, A.V. Chinak, Thermophys. Aeromech. 20, 391 (2013) 